\title{
SINTOMAS OSTEOMUSCULARES EM FISIOTERAPEUTAS E ENFERMEIROS NO AMBIENTE HOSPITALAR
}

\author{
Carolina Barbosa da Silva*, Caroline Souza Andrade Rocha**, Márcio Massao Kawano***, \\ Mansueto Gomes Neto****, Bruno Prata Martinez***** \\ Autor correspondente: Bruno Prata Martinez - brunopmartinez@hotmail.com \\ * Fisioterapeuta graduada pela Faculdade Social da Bahia - FSBA, Salvador, BA - Brasil. arolinabsilva2010@hotmail.com \\ ** Fisioterapeuta graduada pela Faculdade Social da Bahia - FSBA, Salvador, BA - Brasil. k_rol_rocha@hotmail.com \\ *** Fisioterapeuta, doutorando em Medicina e Saúde Humana pela Escola Bahiana de Medicina e Saúde Pública. Docente \\ da Faculdade São Francisco de Barreiras, Barreiras, BA - Brasil. marcio_kawano@yahoo.com.br \\ **** Fisioterapeuta, doutor em Medicina e Saúde pela UFBA e mestre em Ciências da Reabilitação pela UFMG. Docente da \\ Universidade Federal da Bahia, Salvador, BA - Brasil. netofisio@gmail.com \\ ***** Fisioterapeuta, mestrando em Medicina e Saúde pela Escola Bahiana de Medicina e Saúde Pública, professor \\ da Faculdade Social da Bahia e professor auxiliar da Universidade do Estado da Bahia (UNEB), Salvador, BA - Brasil. \\ brunopmartinez@hotmail.com
}

\section{Resumo}

Objetivo: Descrever os sintomas osteomusculares em fisioterapeutas e enfermeiros em um hospital na cidade de Salvador-Bahia. Metodologia: Trata-se de um estudo transversal, realizado entre agosto e novembro de 2010, utilizado como instrumento o Questionário Nórdico de Sintomas Osteomusculares. Outras variáveis avaliadas foram carga horária semanal e tempo de atuação profissional. Resultados: Dos 76 profissionais avaliados, 43 eram fisioterapeutas e 33 enfermeiros, com idade média de $28,6 \pm 4,4$ anos e IMC 24,1 $\pm 5,4 \mathrm{~kg} / \mathrm{m}^{2}$. O tempo de atuação foi $3,5 \pm 4,1$ anos e a jornada de trabalho semanal 46,8 $\pm 18,5$ horas. Houve predomínio de sintomas osteomusculares na região lombar $(60,5 \%)$ e no dorso do tórax $(46,5 \%)$ em fisioterapeutas e pescoço $(60,6 \%)$ e ombros $(54,5 \%)$ nos enfermeiros. As mulheres apresentaram maior ocorrência de que em relação aos homens no pescoço $(57,1 \%$ vs $33,3 \% ; p=0,047$ ) e nos ombros ( $49 \%$ vs 14,8\%; $p=0,003$ ). Os enfermeiros tiveram maior ocorrência de queixas nos ombros $(54,5 \%$ vs $23,3 \%$; $p=0,005)$ e quadril $(24,2 \%$ vs $7 \% ; p=0,034)$, do que os fisioterapeutas sendo que os profissionais com jornada superior a 44 horas semanais tiveram maiores repercussões nas atividades de vida diária (12,9\% vs $0 \% ; p=0,013)$. Conclusão: A frequência de sintomas nos enfermeiros e fisioterapeutas no ambiente hospitalar foi elevada nas regiões lombar, ombros, punhos e cervical, provavelmente devido as atividades laborais e posturas inadequadas. Outro dado preocupante é o pequeno tempo de atuação profissional, o que sugere a necessidade de realização de medidas preventivas para não evolução e agravamento dos sintomas.

Palavras-chave: Transtornos Traumáticos Cumulativos; Fisioterapeutas; Enfermeiros; Hospital. 


\title{
SYMPTOMS IN MUSCULOSKELETAL PHYSIOTHERAPISTS AND NURSES IN THE HOSPITAL SETTING
}

\begin{abstract}
Purpose: To describe the musculoskeletal symptoms in physiotherapists and nurses in a hospital in the city of Salvador, Bahia. Methods: This was a cross-sectional study, conducted between august and november 2010, using as instrument the Nordic Musculoskeletal Questionnaire. Other variables were assessed workload and time of practice. Results: Of the 76 professional reviews, 43 were physical therapists and 33 nurses with a mean age of $28.6 \pm 4.4$ years, BMI $24.1 \pm 5.4 \mathrm{~kg} / \mathrm{m}^{2}$. The time of practice was $3.5 \pm 4.1$ years and the workweek $46.8 \pm 18.5$ hours. There was a prevalence of musculoskeletal symptoms in the lower back $(60.5 \%)$ and back $(46.5 \%)$ in physical therapists and neck $(60.6 \%)$ and shoulder $(54.5 \%)$ in nurses. Women were more frequent compared to men in the neck ( $57.1 \%$ vs $33.3 \%, p=0.047$ ) and shoulder ( $49 \%$ vs $14.8 \%, p=0.003)$. The nurses had a higher occurrence of problems that physiotherapists shoulders $(54.5 \%$ vs $23.3 \%, p=0.005)$ and hip $(24.2 \%$ vs $7 \%, p=0.034)$, and professionals with journey more than 44 hours per week had greater impact on activities of daily living (12.9\% vs $0 \%, p=0.013)$. Conclusion: The frequency of symptoms in nurses and physical therapists in the hospital was higher in the lumbar region, shoulders, wrists and neck, probably due to work activities and postures. Another disturbing finding is the small time of practice, which suggests the need for carrying out preventive measures for not evolving and worsening of symptoms.
\end{abstract}

Keywords: Cumulative Trauma Disorders; Physiotherapists; Nurses; Hospital.

\section{INTRODUÇÃO}

A síndrome relacionada ao trabalho é caracterizada pelo conjunto de disfunções musculoesqueléticas na coluna, membro superior e inferior, gerando dor, parestesia, fadiga e fraqueza. Esse conjunto de disfunções é denominado de Lesão por Esforço Repetitivo/ Distúrbios Osteomusculares Relacionados ao Trabalho (LER/DORT). . $^{(1,2)}$

A LER/DORT pode ser considerada como um problema de saúde pública, devido às suas repercussões na vida dos trabalhadores que prestam assistência à saúde. Profissionais de saúde que atuam em ambiente hospitalar lidam no seu dia-a-dia com indivíduos acamados que necessitam de auxílio nas suas atividades de vida diária, sendo expostos a grande sobrecarga, portanto, esses pro- fissionais compõe um grupo de risco para essas lesões. ${ }^{(3)}$

Dados epidemiológicos confirmam uma alta prevalência das disfunções osteomusculares em profissionais de saúde como fisioterapeutas e enfermeiros. Em 2005, Murofuse e Marziale observaram que dos 6.070 atendimentos realizados aos trabalhadores de enfermagem, houve predominância das condições relacionadas aos problemas osteomusculares $(11,8 \%)$, sendo que $58,4 \%$ foram agrupados em dorsopatias, seguidos pelos transtornos de tecidos moles $(39,6 \%)$. (4) Costa et al. (2009) identificaram uma prevalência de dor crônica entre $13 \%$ e ou a $53 \%$ em enfermeiras de um 
hospital escola, sendo maior no gênero feminino e a sua prevalência aumentando com a idade. ${ }^{(3)}$

No estudo de Alrowayeh et al. (2010), envolvendo 350 fisioterapeutas do Kuwait, foi observado $47,6 \%$ de desordens osteomusculares, com predomínio de dor lombar (32\%). (5) Já no estudo de Siqueira et al. (2008), realizado no Brasil com fisioterapeutas, a ocorrência de desordens osteomusculares na região lombar foi de $78,5 \%$.(6)

Dificuldades no setor da saúde, tarefas peculiares dos profissionais e escassez de recursos humanos são condições de trabalho vividas pelos profissionais de saúde, que contribuem para o desenvolvimento de LER/DORT. ${ }^{(7,8)}$

Profissionais de saúde podem apresentar desgaste físico, emocional, os quais refletem negativamente na qualidade do serviço prestado.(9)

Assim, tanto as atividades laborais como as atividades da vida diária, podem gerar sobrecarga em curto ou a longo prazo, sendo em algumas situações responsáveis por uma porcentagem significativa da queda do desempenho no trabalho. ${ }^{(10,11)}$

As alterações osteomusculares podem gerar incapacidade laboral e absenteísmo em profissionais de saúde e sua identificação de forma precoce pode contribuir no desenvolvimento de estratégias de prevenção e tratamentos de saúde. Desta forma, o presente estudo teve como objetivo descrever os sintomas osteomusculares em fisioterapeutas e enfermeiros no ambiente hospitalar em um hospital público na cidade de Salvador-Bahia.

\section{MATERIAL E MÉTODO}

Este estudo descritivo e transversal foi realizado no hospital Irmã Dulce - Obras Sociais Irmã Dulce, na cidade de Salvador-Bahia, no período compreendido entre outubro a dezembro/2010. Os critérios de inclusão do presente estudo foram fisioterapeutas e enfermeiros que trabalhassem com pacientes adultos no referido hospital, com idade superior a 18 anos e que aceitassem participar da pesquisa através da assinatura do termo de consentimento livre e esclarecido. $O$ estudo foi aprovado pelo comitê de ética do Hospital, sob protocolo número 44/10.

A coleta de dados foi iniciada pela compilação de informações sócio-demográficas como idade, sexo, índice de massa corporal (IMC), estado civil, profissão, tempo de atuação profissional, jornada de trabalho e carga horária profissional através de questionário elaborado pelos autores da pesquisa.

Para avaliação da frequência dos sintomas osteomusculares foi aplicado o (QNSO), ${ }^{(12,13)}$ o qual é composto por escolhas binárias relacionadas a ocorrência de sintomas em determinadas regiões corporais, sendo considerado os 12 meses e os sete dias precedentes à entrevista, sendo relatado também o afastamento das atividades no último ano. Esse instrumento foi validado para população brasileira, sendo considerado como um bom índice de morbidade osteomuscular. ${ }^{(12)}$

Para análise dos dados demográficos e clínicos, foram utilizadas estatísticas descritivas. Os dados de variáveis contínuas foram descritos em médias e desvio-padrão (idade, IMC, tempo de serviço e de jornada de trabalho) e as categóricas foram descritas em medidas de freqüência e expressos como porcentagens (respostas do QNSO, sexo e profissão). A análise intergrupos para sexo, presença de obesidade e tipo de profissão (fisioterapeutas e enfermeiros) foi feita em relação aos quatro domínios do questionário, ao tempo de serviço e de atuação profissional através do teste Qui-quadrado. O tempo de serviço foi estratificado em dois grupos, sendo um com menos que cinco anos e o outro com mais de cinco anos de atuação. Já a carga horaria semanal foi dividida em: com mais de 44 horas e com menos de 44 horas por semana. O nível de significância foi um valor de $p$ menor que $5 \%(0,05)$. O software utilizado foi o Statistical Package for the Social Sciences (SPSS) for Windows (versão 14.0).

\section{RESULTADOS}

A amostra foi composta por 76 profissionais de saúde, com uma média de idade de 28,6 $\pm 4,4$ anos e IMC de $24,1 \pm 5,4 \mathrm{~kg} / \mathrm{m}^{2}$. Do total de indivíduos 
avaliados, 43 eram fisioterapeutas $(56,6 \%)$ e 33 eram enfermeiros $(43,4 \%)$, existindo predomínio do sexo feminino (64,5\%). Quanto à descrição dos dados sociodemográficos, os resultados mostraram que em relação ao estado civil houve predomi- nância dos solteiros $(71,1 \%)$, seguido dos casados $(23,7 \%)$. A média de tempo de atuação profissional foi de 3,5 $\pm 4,1$ anos, sendo a média de jornada de trabalho semanal igual a $46,8 \pm 18,5$ horas/semana. Os dados estão descritos na Tabela 1.

Tabela 1 - Características sóciodemográficas dos 76 profissionais de saúde avaliados no hospital Santo Antônio Obras Sociais Irmã Dulce, na cidade de Salvador-Bahia, 2010.

\begin{tabular}{|c|c|c|c|}
\hline CaRActerística & CATEGoRIA & MÉDIA \pm DP & $N(\%)$ \\
\hline Idade (anos) & ---- & $28,6 \pm 4,4$ & ---- \\
\hline IMC $\left(\mathrm{kg} / \mathrm{m}^{2}\right)$ & --- & $24,1 \pm 5,4$ & --- \\
\hline Tempo atuação profissional (anos) & --- & $3,5 \pm 4,1$ & ---- \\
\hline $\begin{array}{c}\text { Carga horária semanal } \\
\text { (horas) }\end{array}$ & --- & $46,8 \pm 18,5$ & ---- \\
\hline \multirow[t]{2}{*}{ Profissão } & Fisioterapeutas & --- & $43(56,6)$ \\
\hline & Enfermeiros & --- & $33(43,4)$ \\
\hline \multirow[t]{2}{*}{ Sexo } & Masculino & --- & $27(35,5)$ \\
\hline & Feminino & ---- & $49(64,5)$ \\
\hline \multirow[t]{3}{*}{ Estado civil } & Solteiro & --- & $54(71,1)$ \\
\hline & Casado & --- & $18(23,7)$ \\
\hline & Divorciado & --- & $4(5,2)$ \\
\hline
\end{tabular}

Na Tabela 2 estão descritos a frequência de ocorrência de problemas como dor, formigamento e dormência, nos últimos 12 meses em diferentes regiões anatômicas, no qual se pode observar que a prevalência dessas alterações ocorreram em diferentes regiões anatômicas quando foram comparadas as duas profissões. As maiores ocorrências de alterações osteomusculares nos fisioterapeutas foram na região lombar $(60,5 \%)$, dorso do tórax (costas) $(46,5 \%)$ pescoço e punhos $(30,2 \%)$, diferentemente dos enfermeiros que apresentaram maiores ocorrências na região do pescoço $(60,6 \%)$, nos ombros (54,5\%), na região lombar $(48,5 \%)$ e nos tornozelos $(42,4 \%)$. Na análise intergrupo, foi identificado que as mulheres apresentaram uma maior ocorrência de queixa em relação aos homens no pescoço $(57,1 \%$ vs $33,3 \%$; $p=0,047)$ e nos ombros ( $49 \%$ vs $14,8 \% ; p=0,003$ ). Foi visto também, que os enfermeiros tiveram maior ocorrência de problemas do que os fisioterapeutas nos ombros ( $54,5 \%$ vs $23,3 \% ; p=0,005)$, no quadril $(24,2 \%$ vs $7 \% ; p=0,034)$ e nos tornozelos $(42,4 \%$ vs $18,6 \%$; $\mathrm{p}=0,023)$. Não foi observado diferença entre o tempo de serviço e jornada semanal. 
Tabela 2 - Características dos sintomas osteomusculares relacionados ao trabalho nos últimos 12 meses por região, referidos pelos profissionais do hospital citado, Salvador-Bahia,2010

\begin{tabular}{|c|c|c|c|c|}
\hline REGIÃO ANATÔMICA & FISIOTERAPEUTAS & $(N=43)$ & ENFERMEIROS & $N=33$ \\
\hline Pescoço & 17 & $39,5 \%$ & 20 & $60,6 \%$ \\
\hline Ombros & 10 & $23,3 \%$ & 18 & $54,5 \%$ \\
\hline Costas & 20 & $46,5 \%$ & 13 & $39,4 \%$ \\
\hline Cotovelos & 3 & $7,0 \%$ & 1 & $3,0 \%$ \\
\hline Punhos/Mãos & 13 & $30,2 \%$ & 11 & $33,3 \%$ \\
\hline Lombar & 26 & $60,5 \%$ & 16 & $48,5 \%$ \\
\hline Quadril/Coxas & 3 & $7,0 \%$ & 8 & $24,2 \%$ \\
\hline Joelhos & 11 & $25,6 \%$ & 13 & $39,4 \%$ \\
\hline Tornozelos & 8 & $18,6 \%$ & 14 & $42,4 \%$ \\
\hline
\end{tabular}

Diante dos resultados obtidos, o presente estudo evidenciou que os sintomas osteomusculares referidos não tiveram um grande impacto nas atividades normais como trabalho, lazer ou atividade doméstica, como demonstrado pelos valores percentuais descritos na Tabela 3. A maior causa de afastamento das atividades para os fisioterapeutas foram as alterações nos tornozelo $(16,3 \%)$, seguido das alterações na região lombar $(11,6 \%)$. Já na população de enfermeiros participantes do estudo, a região lombar $(15,2 \%)$, seguida do pescoço $(12,1 \%)$ foram as que mais levaram estes profissionais a se afastarem das suas atividades normais. Em relação ao gênero, os homens apresentaram como maiores causas de afastamento os joelhos $(14,8 \%$ vs $0 \%$; $p=0,006)$ e os tornozelos $(25,9 \%$ vs $4,1 \%$; $\mathrm{p}=0,005)$. Os profissionais que tiveram uma carga horaria maior que 44 horas semanais também tiveram maiores queixas nesse domínio do questionário $(12,9 \%$ vs $0 \% ; p=0,013)$. Não houve diferença entre o tipo de profissão e tempo de serviço.

Tabela 3 - Descrição da quantidade de profissionais que referiram que nos últimos 12 meses, foram impedidos (a) de realizar atividades normais (Exemplo: trabalho, atividades domésticas e de lazer) por causa desse problema, SalvadorBahia, 2010

\begin{tabular}{ccccc}
\hline Região anAtÔMICA & FisioterapeUtAS & $\mathbf{N = 4 3}(\%)$ & ENFERMEIROS & N=33 (\%) \\
\hline Pescoço & 3 & $7,0 \%$ & 4 & $12,1 \%$ \\
Ombros & 2 & $4,7 \%$ & 2 & $6,1 \%$ \\
Costas & 2 & $4,7 \%$ & 3 & $9,1 \%$ \\
Cotovelos & 0 & $0 \%$ & 0 & $0 \%$ \\
Punhos/Mãos & 1 & $2,3 \%$ & 2 & $6,1 \%$ \\
Lombar & 5 & $11,6 \%$ & 5 & $15,2 \%$ \\
Quadril/Coxas & 2 & $4,7 \%$ & 1 & $3,0 \%$ \\
Joelhos & 3 & $7,0 \%$ & 1 & $3,0 \%$ \\
Tornozelos & 7 & $16,3 \%$ & 2 & $6,1 \%$ \\
\hline
\end{tabular}


Já a Tabela 4 descreve as alterações que levaram os participantes a consultarem algum profissional de saúde, como médico ou fisioterapeuta nos últimos 12 meses. A alteração mais prevalente que levou os fisioterapeutas a procurarem assistência de saúde foram as regiões tornozelo $(16,3 \%)$, pescoço $(16,3 \%)$ e região lombar $(11,3 \%)$, diferentemen- te dos enfermeiros os quais procuraram essas assistências devido as alterações em região lombar e em costas (18,2\%), seguidas do pescoço, ombro, tornozelo que apresentaram o mesmo percentual $(12,1 \%)$. Não foi observado diferença entre o tipo de profissão, tempo de serviço e jornada semanal.

Tabela 4 - Descrição do percentual de profissionais de saúde do referido hospital que relataram ter consultado algum profissional da área de saúde nos últimos 12 meses por causa dos sintomas osteomusculares citados, Salvador-Bahia, 2010.

\begin{tabular}{ccccc}
\hline Região anatômica & Fisioterapeutas & $\mathbf{N = 4 3}(\%)$ & ENFERMEIROS & $\mathbf{N = 3 3}(\%)$ \\
\hline Pescoço & 7 & $16,3 \%$ & 4 & $12,1 \%$ \\
Ombros & 4 & $9,3 \%$ & 4 & $12,1 \%$ \\
Costas & 4 & $9,3 \%$ & 6 & $18,2 \%$ \\
Cotovelos & 0 & $0 \%$ & 1 & $3 \%$ \\
Punhos/Mãos & 1 & $2,3 \%$ & 2 & $6,1 \%$ \\
Lombar & 5 & $11,6 \%$ & 6 & $18,2 \%$ \\
Quadril/Coxas & 3 & $7 \%$ & 2 & $6,1 \%$ \\
Joelhos & 3 & $7 \%$ & 2 & $6,1 \%$ \\
Tornozelos & 7 & $16,3 \%$ & 4 & $12,1 \%$ \\
\hline
\end{tabular}

O aparecimento de distúrbios osteomusculares nos últimos 7 dias que antecederam a pesquisa também foi evidenciado no estudo, a qual foi descrito na Tabela 5. Nos enfermeiros as alterações mais frequentemente relatadas foram a região lombar $(25,6 \%)$, tornozelos $(15,2 \%)$, pescoço e ombros
$(12,1 \%)$. Nos fisioterapeutas as regiões mais acometidas foram lombar $(25,6 \%)$, pescoço $(20,9 \%)$, dorso de tórax $(16,3 \%)$ e joelhos $(16,3 \%)$. Não foi observado diferença entre o tipo de profissão, tempo de serviço e jornada semanal.

Tabela 5 - Características dos sintomas osteomusculares relacionados ao trabalho nos últimos 7 dias por região, referidos pelos profissionais $(n=76)$, Salvador-Bahia, 2010.

\begin{tabular}{ccccc}
\hline Região anatômica & Fisioterapeutas & $\mathbf{N = 4 3}(\%)$ & ENFERMEIROS & $\mathbf{N = 3 3}$ (\%) \\
\hline Pescoço & 9 & $20,9 \%$ & 4 & $12,1 \%$ \\
Ombros & 6 & $14,0 \%$ & 4 & $12,1 \%$ \\
Costas & 7 & $16,3 \%$ & 2 & $6,1 \%$ \\
Cotovelos & 0 & $0 \%$ & 1 & $3,0 \%$ \\
Punhos/Mãos & 5 & $11,6 \%$ & 3 & $9,1 \%$ \\
Lombar & 11 & $25,6 \%$ & 8 & $24,2 \%$ \\
Quadril/Coxas & 3 & $7,0 \%$ & 0 & $0 \%$ \\
Joelhos & 7 & $16,3 \%$ & 3 & $9,1 \%$ \\
Tornozelos & 6 & $14 \%$ & 5 & $15,2 \%$ \\
\hline
\end{tabular}


Não houve diferença nos quatros domínios do questionário na análise intergrupo para obesos e não-obesos.

\section{DISCUSSÃO}

O presente estudo identificou uma alta ocorrência de alterações osteomusculares nos enfermeiros e fisioterapeutas no ambiente hospitalar, o qual foi semelhante as altas prevalências em outros grupos profissionais descritas na literatura. ${ }^{(10,14-21)}$ Dentre os participantes do estudo houve predominância do sexo feminino, o que corrobora com outros estudos sobre os esses profissionais de saúde, (3,4,6,622-28) cujos sintomas musculoesqueléticos são justificados pela elevada jornada de trabalho, além da associação com as tarefas domésticas. Estes resultados refletem dados históricos, onde a força de trabalho na saúde tem se configurado eminentemente pelo sexo feminino.

Os fisioterapeutas relataram um maior percentual de sintomas osteomusculares na região lombar $(60,5 \%)$, o que corrobora com outros estu$\operatorname{dos}^{(5,6,26,29)}$ que identificaram uma alta prevalência de lombalgias em fisioterapeutas (32\%; $78,6 \%$; $50,8 \%$ e $63 \%$ respectivamente). Houve também altos percentuais de sintomas na região das costas $(46,5 \%)$, do pescoço e dos punhos $(30,2 \%)$, o que se assemelha a outros estudos. ${ }^{(5,26)}$ Uma possível justificativa para isso, pode ser a atividade laboral na postura ortostática, com o tronco fletido para assistência aos pacientes acamados. Assim, o fisioterapeuta pode ser considerado um profissional de risco para o aparecimento de alterações osteomusculares, por causa das atividades com sobrecarga de peso e as posturas inadequadas, devendo-se atentar para melhores posturas e adaptações para diminuição da sobrecarga para o seu corpo.

Em relação aos enfermeiros avaliados, também existiu uma elevada ocorrência de problemas osteomusculares, destacando-se as regiões do pescoço $(60,6 \%)$, dos ombros $(54,5 \%)$ e lombar $(48,5 \%)$. Esses dados convergem com outro estudo, (4) na qual foi evidenciando que $43 \%$ dos enfermeiros apresentaram alterações osteomusculares e do tecido conjuntivo, sendo que as artropatias (16,6\%), as dorsopatias $(50,7 \%)$ e os transtornos dos tecidos moles $(27,5 \%)$, representaram quase que a totalidade $(94,8 \%)$ das doenças do sistema osteomuscular (DSOM). Essa ocorrência mostra que a população de enfermeiros apresenta susceptibilidade ao desenvolvimento de problemas osteomusculares, devendo existir uma maior atenção para esta população, para não evolução para um quadro de incapacidade laboral.

Apesar dos trabalhadores de enfermagem apresentarem um alto risco de adquirirem alterações osteomusculares, outro estudo, (3) descreveu que os enfermeiros foram responsáveis por apenas 13\% dos 76 afastamentos por alterações osteomusculares, o que se assemelha com o presente estudo, que também mostrou um pequeno porcentagem de absenteísmo. Entretanto, uma justificativa para isso é o fato dos profissionais terem uma baixa média de idade e curto tempo de atuação profissional, o que aumenta sua resistência, porém não evita a ocorrência de maiores complicações no futuro.

No presente estudo a média de idade dos participantes foi baixa $(28,6 \pm 4,4$ anos), apesar do alto índice de alterações osteomusculares identificadas, o que sugere um aparecimento precoce de agravos á saúde destes trabalhadores, divergindo de outros estudos, que encontraram uma maior ocorrência das alterações nos indivíduos com idades mais elevadas. $(5,6,24-25,30)$ Esse dado sugere uma maior preocupação com esses profissionais de saúde e sua atividade laboral, já que os sintomas já estão presentes mesmo numa idade precoce.

Outros estudos envolvendo outros profissionais em saúde também ratificam essa alta prevalência de problemas osteomusculares, justificando a necessidade de intervenções preventivas para não agravamento. $\mathrm{Na}$ população de cirurgiões-dentistas, por exemplo, os resultados obtidos indicaram que $89,1 \%$ dos pesquisados tinham experimentado algum sintoma de LER/DORT nos últimos 12 meses, sendo a região lombar, o pescoço e o ombro as regiões mais acometidas, provavelmente pela 
utilização de instrumentos que não obedecem a critérios ergonômicos e a falta de atenção com a postura. ${ }^{(10)}$ Já outro estudo, envolvendo médicos ultrassonografistas, também identificou que estes profissionais são bastante acometidos por alterações osteomusculares, as quais foram relatadas em $85 \%$ dos profissionais. ${ }^{(18)}$ As principais causas relatadas foram alta carga diária, postura estática com realização de micromovimentos e tempo de duração de exames realizados.

Em relação a carga horária semanal de trabalho, verificou-se no presente estudo, que os indivíduos com um período superior a 44 horas apresentaram uma maior frequência de afastamento para atividades do dia-a-dia secundário a dores $(12,9 \%$ vs $0 \%$; $p=0,014)$, o que sugere uma possível influência da atividade laboral nos sistemas osteomusculares. Já o tempo de atuação profissional também foi baixo (3,5 $\pm 4,13$ anos), quando comparado a outros estudos que também relataram alta prevalência de sintomas. ${ }^{(6,24,25)}$

Essa alta frequência de sintomas de forma precoce é um dado preocupante, já que o tempo necessário para aposentadoria na população geral é 30 anos para o sexo feminino e 35 para o sexo masculino. Dentre alguns prováveis fatores desencadeantes é possível citar a força excessiva e as atividades repetitivas realizadas, na maioria das vezes em posturas inadequadas, durante a prática laboral do cuidado no ambiente hospitalar. Entretanto as taxas de afastamentos foram baixas, provavelmente pelo curto tempo de exposição e idade dos profissionais, bem como a procura por profissionais para o tratamento dos sintomas. Quanto à presença de queixas nos últimos sete dias, a região lombar foi a mais acometida entre os fisioterapeutas e enfermeiros, corroborando estudo prévio. ${ }^{(23)}$

Dentre as limitações do estudo, pode ser citado o tamanho amostral, o qual não foi maior devido o estudo ter sido realizado em apenas um hospital. Outra limitação foi não ter sido coletado o nível de atividade física desses indivíduos, já que este pode ser um fator protetor para as alterações osteomusculares.

\section{CONCLUSÃO}

A frequência de sintomas osteomusculares é elevada em enfermeiros e fisioterapeutas no ambiente hospitalar, principalmente nas regiões lombar, nos ombros, punhos e região cervical, provavelmente devido as atividades laborais com sobrecarga de peso e posturas inadequadas. Outro dado preocupante é o pequeno tempo de atuação profissional, o que sugere a necessidade de realização de medidas preventivas e ergonômicas para não evolução e agravamento dos sintomas.

\section{REFERÊNCIAS}

1. Chiavegato FG, Pereira JRA. LER/DORT: multifatorialidade etiológica e modelos explicativos. Interface comun. saúde educ. 2004;8(14):149-162.

2. Strazdins L, Bammer G. Women, work and musculoskeletal health. Soc Sci Med. 2004;58(6):997-1005.

3. Costa FM, Vieira MA, Sena RR. Absenteísmo relacionado à doenças entre membros da equipe de enfermagem de um hospital escola. Rev. bras. enferm. 2009;62(1):38-44.

4. Murofuse NT, Marziale, MHP. Doenças do sistema osteomuscular em trabalhadores de enfermagem. Rev Lat Am Enfermagem. 2005;13(3):364-73.

5. Alrowayeh HN, Alshatti TA, Aljadi SH, Fares M, Alshamire MM, Alwazan SS. Prevalence, characteristics, and impact of work-related musculoskeletal disordes:a survey among physical therapists in the States of Kuwait. BMC Musculoskelet Disord. 2010;11(116):1-11.

6. Siqueira GR, Cahú FGM, Vieira RAG.

Ocorrência de lombalgias em fisioterapeutas na cidade de Recife, Pernambuco. Rev. bras. fisioter. 2008;12(3):222-227.

7. Waters TR. National efforts to identify research issues related to prevention work-related musculoskeletal disorders. J Electromyogr Kinesiol. 2004;14(1):7-12. 
8. Royas AD, Marziale MHP. A situação de trabalho do pessoal de enfermagem no contexto de um hospital argentino: um estudo sob a ótica da ergonomia. Rev Lat Am Enfermagem. 2001; 9(1):102-108.

9. Sancinetti TR et al. Absenteísmo - doença na equipe de enfermagem: relação com a taxa de ocupação. Rev. Esc. Enferm. USP. 2009;43(2):1277-83.

10. Regis FGI, Michels G, Sell I. Lesões por esforços repetitivos/distúrbios osteomusculares relacionados ao trabalho de cirurgiõesdentistas: aspectos biomecânicos. Produção. 2009;19(3):569-580.

11. Carneiro RLV, Coqueiro RS, Freire MO, Barbosa AR. Sintomas de distúrbios osteomusculares em motoristas e cobradores de ônibus. Rev. bras. cineantropom. desempenho hum. 2007;9(3):277283.

12. Pinheiro FA, Troccoli BT, Carvalho CV. Validação do Questionário Nórdico de Sintomas Osteomusculares como medida de morbidade. Rev. saúde pública. 2002;36(3):307-312.

13. Barros EMC, Alexandre NMC. Cross-cultural adaptation of the Nordic musculoskeletal questionnaire. Int Nurs Rev. 2003;50(2):101108.

14. Santos FS, Barreto S. Atividade ocupacional e prevalência de dor osteomuscular em cirurgiões dentistas de Belo Horizonte, Minas Gerais, Brasil: contribuição ao debate sobre os distúrbios osteomusculares relacionados ao trabalho. Cad. saúde pública. 2001;17(1):181-93.

15. Brandão AG, Horta BL, Tomasi E. Sintomas de distúrbios osteomusculares em bancários de Pelotas e região: prevalência de fatores associados. Rev. bras. epidemiol. 2005;8(3):295-305.

16. Picoloto $D$, Silveira E. Prevalência de sintomas osteomusculares e fatores associados em trabalhadores de uma industria metalúrgica de Canoas - RS. Ciênc. saúde coletiva. 2008;13(2):507-516.

17. Rocha LE, Ribeiro DM. Trabalho, saúde e gênero: estudo comparativo sobre analistas de sistemas. Rev. saúde pública. 2001;35(6):539-47.
18. Barbosa LH, Coury HJCG. A atividade do médico ultra-sonografista apresenta riscos para o sistema músculo-esquelético? Radiol. bras. 2004;37(3):187-191.

19. Isosaki M, Cardoso E, Glina DMR, Alves ACDC, Rocha LE. Prevalência de sintomas osteomusculares entre trabalhadores de um Serviço de Nutrição Hospitalar em São PauloSP. Rev. bras. saúde ocup. 2011; 36(124):238246.

2O. Martarello NA, Benatti MCC. Qualidade de vida e sintomas osteomusculares em trabalhadores de higiene e limpeza hospitalar. Rev. Esc. Enferm. USP. 2009;43(2):422-8.

21. Vitta AD, Zapater AR, Campos RS, Padovani CR. Desconfortos musculoesqueléticos percebidos em trabalhadores de diferentes faixas etárias, gêneros e ocupações. Fisioter. mov. 2007;2O(1):29-36.

22. Reis RJ, Rocca PF, Silveira AM, Bonilla IML, Giné AN, Martin M. Fatores relacionados ao absenteísmo por doença em profissionais de enfermagem. Rev. saúde pública. 2003;37(5):616-623.

23. Gurgueira GP, Alexandre NMC, Correa FHR. Prevalência de Sintomas músculo-esqueléticos em trabalhadoras de enfermagem. Rev Lat Am Enfermagem. 2003;11(5):608-613.

24. Duran ECM, Cocco MIM .Capacidade para o trabalho entre trabalhadores de enfermagem do pronto-socorro de um hospital universitário. Rev Lat Am Enfermagem. 2004;12(1):43-9.

25. Raffone AM, Hennington EA. Avaliação da capacidade funcional dos trabalhadores de enfermagem. Rev. saúde pública. 2005;39(4):669-76.

26. Bagalhi CT, Alqualo-Costa R. Prevalência de distúrbios osteomusculares relacionados ao trabalho em fisioterapeutas. Science in Health. 2011;2(2):93-102.

27. Campo M, Weiser S, Koenig KL, Nordin M. Workrelated musculoskeletal disorders in physical therapists: a prospective cohort study with 1-year follow-up. Phys. ther. 2008; 88(5):608-19.

28. Campo MA, Weiser S, Koenig KL. Job strain in physical therapists. Phys. ther. 2009; 89(9):94656. 
29. Nyland LJ, Grimmer KA. Is undergraduate physiotherapy study a risk factor for low back pain? A prevalence study of LBP in physiotherapy students. BMC Musculoskelet Disord. 2003;9(4):1-12.
30. Pinheiro FA, Tróccoli BT, Paz MGT. Preditores psicossociais de sintomas osteomusculares: a importância das relações de mediação e moderação. Psicol. reflex. crit. 2006;19(1):142150. 